\title{
Effect of Protein Phosphatase Inhibitors on the Development of Mouse Embryos: Protein Phosphorylation Is Involved in the E-Cadherin Distribution in Mouse Two-Cell Embryos
}

\author{
Yuichi Kawai, ${ }^{*}$ Takaomi Yamaguchi, Takahiro Yoden, Motoki Hanada, and Masaharu Miyake \\ Faculty of Pharmaceutical Sciences, Kobe Gakuin University, Ikawadani, Nishi-ku, Kobe 651-2180, Japan. \\ Received September 6, 2001; accepted November 12, 2001
}

Protein phosphorylation plays many important roles in cell functions and cell differentiation. To clarify the roles of protein phosphorylation in early embryonic development in mice, 2-cell embryos were cultured in the presence of various protein phosphatase inhibitors such as calyculin A, okadaic acid, cyclosporin A, tacrolimus (FK506) and benzyl-phosphonic acid. Calyculin A potently inhibited the 2-cell cleavage to the 4-cell stage. The concentration for $50 \%$ inhibition was $0.26 \mathrm{~nm}$. At the same time, we found that calyculin A-treated 2-cell embryos showed a morula-like shape at a concentration of $2 \mathrm{~nm}$ in $24 \mathrm{~h}$. It is well known that E-cadherin plays a key role in the compaction of late 8-cell stage embryos. In this report, we observed the distribution of E-cadherin protein using anti-E-cadherin antibody with a fluorescence microscope, and also evaluated the relative E-cadherin mRNA content at various stages of embryos by RT-PCR and ABI PRISM 7700 System (a real time PCR apparatus). The fluorescence intensity of E-cadherin increased along with the embryonic development. During the embryonic development from the 2-cell stage to the blastocyst stage, the relative E-cadherin mRNA content greatly increased in a time-dependent manner, while the mRNA did not increase with the addition of calyculin A at the 2-cell stage. Therefore, we observed the localization of the E-cadherin protein in calyculin A-treated embryos with a laser microscope. The distribution pattern of E-cadherin was altered by the addition of calyculin $A$ from a scattered pattern throughout the embryos to a localized pattern at the cell-cell boundary region. These results strongly suggest that the distribution of E-cadherin protein is regulated by protein phosphorylation and/or dephosphorylation.

Key words compaction; calyculin A; real time PCR

Embryonic development requires a variety of information to be relayed via intracellular signaling pathways in blastomeres of the embryos. A number of these signals are transduced into cells through cell surface receptors, which initiate one or more signal transduction cascades and alter the state of phosphorylation of various intracellular molecules through the action of both kinases and phosphatases. Numerous protein kinases and phosphatases have been identified. Serine/ threonine protein phosphatases (PPs), which hydrolyze phosphate ester of phosphoprotein, have been classified into four types: PP1, PP2A, PP2B and PP2C based on their substrate specificity and sensitivity to a defined set of inhibitors and activators. ${ }^{1)}$

It is well known that "compaction" is the first cell adhesion event at the late 8-cell stage or 16-cell stage in mammals. Furthermore, it has been demonstrated that the compaction is mediated by microfilaments, microtubules, ${ }^{2,3)}$ intracellular $\mathrm{Ca}^{2+},{ }^{4)}$ protein kinase $\mathrm{C}^{3,5,6)}$ and E-cadherin. ${ }^{7,8)}$ These reports strongly suggested that the serine/threonine phosphorylation was the main post-translational modification of early embryonic development. Recently, Goval and Alexandre showed that genistein, an inhibitor of protein tyrosine kinase, prevented the 4-cell cleavage to the 8-cell stage but did not prevent a compaction-like morphological change at the 4-cell stage (i.e., premature cell flattening at the 4-cell stage). ${ }^{9)}$ This result strongly suggested that the tyrosine phosphorylation might be concerned with the compaction.

In this paper, to clarify the importance of protein phosphorylation we treated 2-cell mouse embryos with various serine/threonine phosphatase inhibitors including calyculin A, okadaic acid, tacrolimus (FK506) and cyclosporin A and a tyrosine phosphatase inhibitor, benzylphosphonic acid, and observed the early embryonic development after the two-cell stage or the localization of the E-cadherin. We demonstrated here that calyculin A prevented the cleavage from the 2-cell to the 4-cell stage at $2 \mathrm{~nm}$ and caused 2-cell flattening. Our results suggest that the compaction is regulated by protein phosphorylation.

\section{MATERIALS AND METHODS}

Reagents and Stock Solutions Calyculin A, okadaic acid and sodium orthovanadate were obtained from Wako Pure Chemical Industries (Osaka, Japan). Benzylphosphonic acid bis-acetoxymethyl ester (BPA- $\left.\mathrm{AM}_{2}\right)$, monoclonal anti mouse E-cadherin antibody and fluorescein isothiocyanate (FITC)-labeled anti mouse $\operatorname{IgG}(\mathrm{H}+\mathrm{L})$ were from Funakoshi (Tokyo, Japan). Rhodamine-labeled anti mouse IgG $(\mathrm{H}+\mathrm{L})$ was from Chemicon International Inc. (Temecula, U.S.A.) Cyclosporin A was a kind gift from Sandz Pharma AG (Switzerland), FK506 was also a kind gift from Fujisawa Pharmaceutical Co., Ltd. (Osaka, Japan). RT-PCR kit (Ready To Go) was purchased from Amersham Pharmacia Biotech (Buckinghamshire, England). For real time PCR, a set of primers and TaqMan Probe were from PE Applied Biosystems (Branchburg, U.S.A.).

The drugs were diluted in culture medium from the following stock solutions: calyculin A, okadaic acid and BPA-AM $: 20 \mathrm{~mm} /$ dimethyl sulfoxide (DMSO); FK506 and cyclosporin A: $4 \mathrm{~mm} /$ ethyl alcohol; sodium orthovanadate: $20 \mathrm{~mm} / \mathrm{milli}-\mathrm{Q}$ water.

Embryo Collection and Culture in Vitro ICR female mice (6-8 weeks old) were superovulated by the intraperitoneal injection of 5 IU pregnant mare's serum gonadotropin 
(PMSG, Teikokuzoki, Tokyo, Japan) followed $48 \mathrm{~h}$ later by $10 \mathrm{IU}$ human chorionic gonadotropin (hCG, Teikokuzoki, Tokyo, Japan). The female mice were placed with the same strain of male mice overnight, and were checked by a vaginal plug the following morning. Pregnant mice were sacrificed by cervical dislocation, and two-cell embryos were collected at $39-41 \mathrm{~h}$ post hCG injection from the oviduct by flushing with culture medium. To obtain unfertilized eggs, female mice were not mated and instead the cumulus masses were removed from the oviducts $14-15 \mathrm{~h}$ after $\mathrm{hCG}$. Cumulus cells were removed from the eggs by treatment with hyaluronidase and the eggs were washed several times with modified Whitten's medium (m-WM; composed of $\mathrm{NaCl}$ $514 \mathrm{mg}, \mathrm{KCl} 36 \mathrm{mg}, \mathrm{KH}_{2} \mathrm{PO}_{4} 16 \mathrm{mg}$, calcium lactate $-5 \mathrm{H}_{2} \mathrm{O}$ $53 \mathrm{mg}, \mathrm{MgSO}_{4}-7 \mathrm{H}_{2} \mathrm{O} 29 \mathrm{mg}, \mathrm{NaHCO}_{3} 190 \mathrm{mg}$, EDTA-2Na $3.7 \mathrm{mg}$, glucose $100 \mathrm{mg}$, Na-lactate $0.37 \mathrm{ml}$, Na-pyruvate $3.5 \mathrm{mg}$, bovine serum albumin (Sigma Fraction V) $300 \mathrm{mg}$, penicillin $\mathrm{G}$ potassium $8 \mathrm{mg}$ and streptomycin sulfate $5 \mathrm{mg}$ in $100 \mathrm{ml}$ medium). About 20-30 embryos were cultured in $0.2 \mathrm{ml}$ of medium in the presence or absence of protein phosphatase inhibitors under paraffin oil (Nacalai tesque, cell culture grade) under $5 \% \mathrm{CO}_{2}$ in air conditions at $37^{\circ} \mathrm{C}$. At every $12 \mathrm{~h}$ the embryos were observed with a phase contrast microscope (Olympus Type IX70). Under these conditions, most of the embryos were developed to the 4-cell stage in $24 \mathrm{~h}$, and to the morula stage in $48 \mathrm{~h}$ and to the blastocyst stage in $72 \mathrm{~h}$ in the absence of inhibitors. The $\mathrm{IC}_{50}$ value was evaluated from the embryonic developmental data at $72 \mathrm{~h}$ culture in the presence or absence of phosphatase inhibitors.

RT-PCR and Real Time PCR Messenger RNA was prepared from the embryos at various stages with the Quick Prep Micro mRNA purification kit (Pharmacia). In the case of the unfertilized eggs, the eggs were surrounded by cumulus cells. The cumulus cells were dispersed by hyaluronidase treatment (Sigma Type 1-S, 250 unit/ml in culture medium, $10 \mathrm{~min}$ at $37^{\circ} \mathrm{C}$ ) prior to mRNA extraction. The washed embryos were collected in batches of 100 to 200 and added to extraction buffer with $7 \mu \mathrm{g}$ yeast ribosomal RNA (Boehringer Manheim) as carrier and $12.5 \mathrm{pg} / 100$ embryos of rabbit globin mRNA (GIBCO BRL) as internal standard. Purified mRNA from oligo-dT cellulose column was subjected to reverse transcription polymerase chain reaction (RTPCR, ABI 9700) or real time quantitative PCR (ABI PRISM 7700). As a template, mRNAs (equivalent to 5 embryos) were used. PCR entailed 1 cycle for $2 \mathrm{~min}$ at $95^{\circ} \mathrm{C} ; 35$ cycles of $95^{\circ} \mathrm{C}$ for $30 \mathrm{~s}, 59^{\circ} \mathrm{C}$ for $15 \mathrm{~s}, 72^{\circ} \mathrm{C}$ for $30 \mathrm{~s}$; and $72^{\circ} \mathrm{C}$ for $2 \mathrm{~min}$. The PCR products were separated using $2 \%$ agarose gel electrophoresis and visualized with ethidium bromide staining. The ratios of staining intensities for E-cadherin were quantified by computerized densitometry (NIH Image). For relative quantitation of the gene expression, a specific primer set and TaqMan Probe of mouse E-cadherin were used. The amplified E-cadherin cDNAs were evaluated by ABI PRISM 7700. Each analysis was done in a minimum of three separate experiments for the embryos at each developmental stage. To confirm whether the PCR products were Ecadherin or not, the amplified products were subjected to DNA Sequeucer (ABI PRISM 310 Genetic Analyzer).

Indirect Immunofluorescence Prior to immunostaining, each embryo was freed from the zona pellucida by introducing it into acid saline solution $(\mathrm{pH} 2.5$ ) containing $0.1 \%$
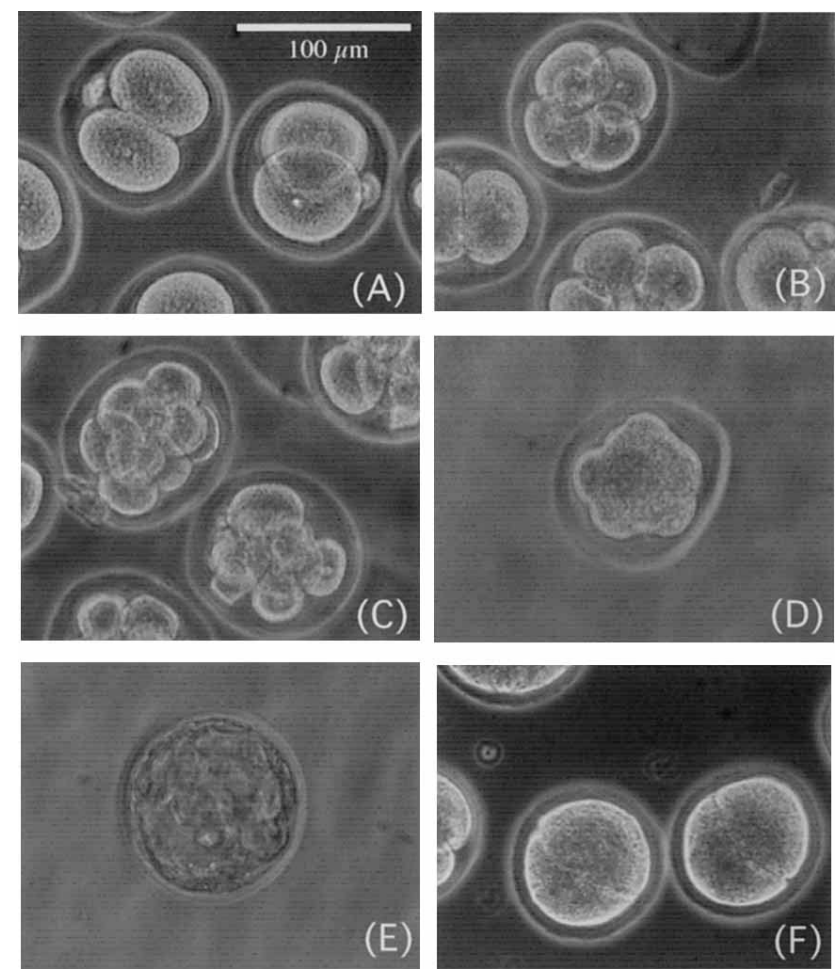

Fig. 1. Photomicrographs of Mouse Preimplantation Embryos

(A) Two-cell, (B) two- to four-cell, (C) six- to eight-cell, (D) compacted morula, (E) expanded blastocyst, (F) two-cell after $24 \mathrm{~h}$ incubation with $2 \mathrm{~nm}$ calyculin A.

polyvinylpyrrolidone. After being washed twice with culture medium, the embryos were fixed with $4 \%$ paraformaldehyde, incubated in the presence of anti mouse E-cadherin antibody $(1: 100)$ for $2 \mathrm{~h}$ at room temperature, and immuno-stained with Rhodamine-labeled anti mouse IgG (1:100) for $60 \mathrm{~min}$ at room temperature under dark conditions. The cell surface E-cadherin content (i.e., red fluorescence) was observed by fluorescence microscope (Olympus, Model VANOX-T).

Laser Microscopy Two-cell embryos were treated with or without $2 \mathrm{~nm}$ calyculin A for $24 \mathrm{~h}$. The embryos were removed from the zona pellucida and fixed with $4 \%$ paraformaldehyde, incubated in the presence of anti mouse E-cadherin antibody for $2 \mathrm{~h}$ at room temperature, and stained with FITC-labeled 2nd antibody for $1 \mathrm{~h}$ at room temperature under dark conditions. The distribution of E-cadherin in the embryos was analyzed by confocal scanning laser fluorescence microscopy (Olympus, Model LSM-GB200).

\section{RESULTS}

The effects of protein phosphatase inhibitors were evaluated at increasing concentrations on at least 60 embryos. Usually, most of the 2-cell stage embryos developed to the 4cell stage at $24 \mathrm{~h}$, to the morula stage (compacted embryo) at $48 \mathrm{~h}$ and to the blastocyst stage at $72 \mathrm{~h}$ in the absence of phosphatase inhibitor (Figs. 1A-E). When 2-cell embryos were cultured in the presence of various inhibitors, the embryonic development was inhibited in a concentration-dependent fashion as shown in Fig. 2. Of these inhibitors, calyculin A was the most powerful inhibitor. Even in the presence of $2 \mathrm{~nm}$ calyculin A, none of the 2-cell embryos developed into 4-cell. In Table 1 , the $50 \%$ inhibitory concentrations of 


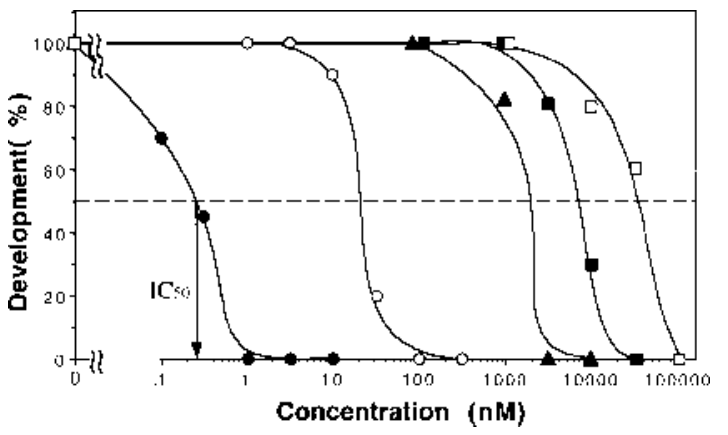

Fig. 2. Development of Mouse Two-Cell Embryos in the Presence of Protein Phosphatase Inhibitors

At least twenty two-cell embryos at each symbol were incubated at $37^{\circ} \mathrm{C}$. Data are expressed as the mean of three independent experiments. The development of mouse embryos was observed with a phase contrast microscope every $12 \mathrm{~h}$. At $72 \mathrm{~h}$-culture, the percentage of development to the blastocyst stage was scored and the $50 \%$ inhibition concentrations of each inhibitor are indicated; calyculin A $(\bullet)$, okadaic acid $(\bigcirc)$,

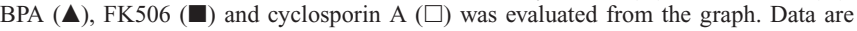
expressed as the mean of three independent experiments.

Table 1. Summary of Phosphatase Inhibitors and $\mathrm{IC}_{50}$ Value

\begin{tabular}{llc}
\hline \hline \multicolumn{1}{c}{ Inhibitors } & Target protein phosphatase & $\mathrm{IC}_{50}(\mathrm{nM})^{a)}$ \\
\hline Calyculin A & Type 1, Type 2A & 0.26 \\
Okadaic acid & Type 2A (Type 1) & 19.30 \\
Benzylphosphonic acid & Tyrosine phosphatase & 2000 \\
FK506 & Type 2B & 8000 \\
Cyclosporin A & Type 2B & 27000
\end{tabular}

a) $\mathrm{The}_{50}$ value means the $50 \%$ inhibition concentration (nм) of the embryonic development in vitro.

these inhibitors $\left(\mathrm{IC}_{50}\right.$ values) are summarized. The protein phosphatase type $2 \mathrm{~B}$ inhibitors (i.e., cyclosporin $\mathrm{A}$ and tacrolimus) and protein tyrosine phosphatase inhibitor (BPA) showed a weak inhibitory activity at the same concentration as the inhibitor of type 1 and/or type $2 \mathrm{~A}$ phosphatase, such as calyculin A and okadaic acid.

Usually, the compaction occurred at the late 8-cell stage. Interestingly, most of the calyculin A-treated 2-cell embryos changed their form into the morula-like shape at $24 \mathrm{~h}$ and their shapes were retained for another $24 \mathrm{~h}$ as shown in Fig. 1F. When 2-cell embryos were treated with $40 \mathrm{~nm}$ of okadaic acid, a few embryos also changed their shape into a morulalike feature. No morphological change was observed in the presence of the other phosphatase inhibitors used (data not shown).

It is well known that the compaction is mediated by E-cadherin molecules. So, the E-cadherin mRNA level was analyzed in calyculin A-treated embryos. Since the level of Ecadherin mRNA in the embryos was very low, RT-PCR procedures with high sensitivity were used. To confirm whether the amplified products were mouse E-cadherin cDNA or not, the amplicons of $414 \mathrm{bp}$ were sequenced with DNA Sequencer. The sequence data perfectly agreed with E-cadherin cDNA (X06115) in Gene Bank database (data not shown). RT-PCR analysis showed that at all stages of the embryos, mRNAs coding for E-cadherin were detected (Fig. 3A). The expression levels of E-cadherin mRNA were compared at various stages of the embryos using NIH Image software, and the results are shown in Fig. 3B. The expression levels of E-cadherin mRNA in the embryos increased along with the
(A)

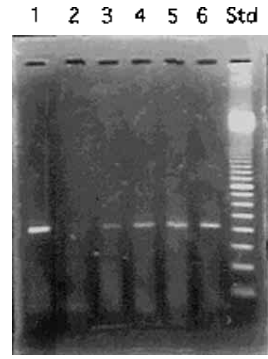

(B)

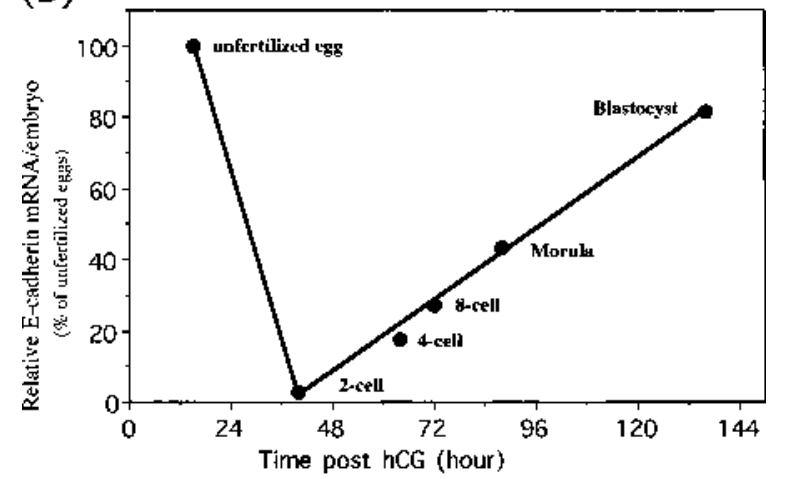

Fig. 3. RT-PCR Analysis of E-Cadherin mRNA at Various Stages of Mouse Embryos

m-RNAs equivalent to five embryos at various stages were amplified by RT-PCR and subjected to agarose gel electrophoresis (A). Each lane represents 1: unfertilized egg, 2: 2-cell, 3: 4-cell, 4: 8-cell, 5: morula, 6: blastocyst, Std: size marker (100 base ladder). The photograph was scanned and relative E-cadherin mRNAs were evaluated by NIH Image software (B).

embryonic development. The transcripts in unfertilized eggs and 2-cell embryos are likely to be maternal mRNA.

To confirm the developmental changes in the E-cadherin mRNA levels during the various stages of the embryo, a real time PCR analysis was conducted using ABI PRISM 7700. A parent solution of mRNA was prepared from unfertilized eggs, and a series of dilutions was conducted by the factor of one fourth, one sixteenth, one sixty-fourth and one two hundred fifty-sixth. A calibration curve was drawn by measuring those diluted solutions. The relative amounts of E-cadherin mRNA at various stages of the embryos were normalized from the curve (data not shown). The relative amounts of Ecadherin m-RNA were as follows: unfertilized eggs, $100 \%$; 2-cell at 0 h-incubation, $18.8 \%$; 4 -cell at $24 \mathrm{~h}, 6.4 \%$; 8 -cell at $36 \mathrm{~h}, 6.5 \%$; morula at $48 \mathrm{~h}, 27.1 \%$; blastocyst at $72 \mathrm{~h}, 74.3 \%$ (Fig. 4A). When 2-cell embryos were incubated with $2 \mathrm{~nm}$ of calyculin A for 3 to $7 \mathrm{~h}$ at $37^{\circ} \mathrm{C}$, the relative amount of Ecadherin mRNA at 3 and $7 \mathrm{~h}$ was $1 / 6$ to $1 / 5$ of 2 -cell, respectively (Fig. 4B).

The localization of E-cadherin protein in the embryos was analyzed with a laser scanning confocal microscope. In normal two-cell embryos, the E-cadherin protein was distributed uniformly throughout the cell surface (Fig. 5A). Usually, 2cell embryos develop to the 4-cell stage in control medium at $24 \mathrm{~h}$. Accordingly, the distribution of E-cadherin protein in the 4-cell embryos was observed. The distribution patterns of E-cadherin in 4-cell embryos were similar to those in 2-cell embryos (data not shown). In contrast, it was localized at the cell-cell apposed regions in the calyculin A-treated embryos (Fig. 5B). Thus, the distribution pattern of E-cadherin protein 
(A)

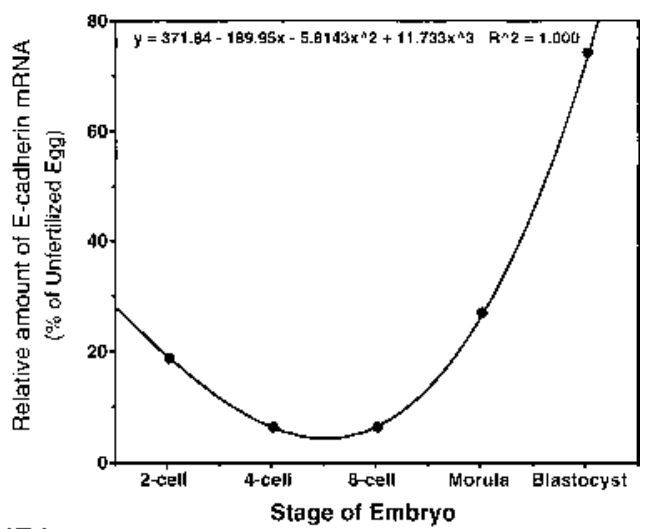

(B)

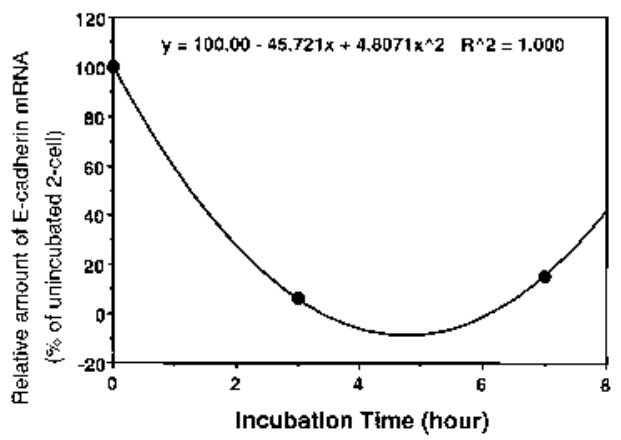

Fig. 4. (A) Relative E-Cadherin mRNA Contents at Various Stages of Embryos by Real Time PCR (ABI PRISM 7700)

mRNAs from various stages of mouse embryos were amplified by real time PCR The relative mRNA content at each stage of the mouse embryo was normalized to the unfertilized egg's E-cadherin mRNA content. The data represent the $\%$ of unfertilized eggs.

(B) Relative E-Cadherin mRNA Contents in Two-Cell Embryos before or after Calyculin A-Treatment

Two-cell embryos were cultured for 3 to $7 \mathrm{~h}$ in the presence of $2 \mathrm{~nm}$ calyculin A. The mRNAs were extracted from the embryos and relative amounts of E-cadherin mRNA were estimated by real time PCR. The data were normalized to mRNA content of twocell embryos before incubation with calyculin A.

\section{(A) Control 2-cell}
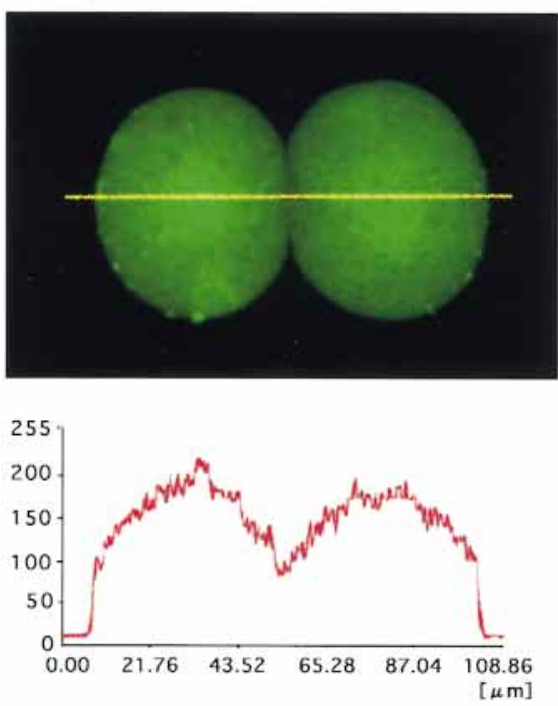

was altered by calyculin A treatment.

\section{DISCUSSION}

Embryos require a variety of intracellular signaling pathways for their development. A number of these signals are transduced through the action of both protein kinases and phosphatases. Numerous protein kinases and phosphatases are known. Smith et al. reported that PP1 and PP2A isozymes among many phosphatases existed specifically in mouse oocytes. ${ }^{10)}$ Furthermore, the expression and stage-specific localization of protein kinase C (PKC) isotypes during mouse preimplantation development was reported by Pauken and Capco. ${ }^{11)}$

In mice, the phenomenon of compaction happens at the 8cell stage, and the blastomeres which are loosely associated become flattened to each other. The compaction is controlled by post-translational modifications but it does not require protein synthesis, and can be modulated by protein kinase modulators. ${ }^{12)}$ Phorbol esters and diacylglycerides, PKC modulators, can cause a premature compaction at the 2-, 4and early 8-cell stages. ${ }^{3,5)}$ On the other hand, calmodulin antagonists, W5 or W7, ${ }^{5)}$ or PKC inhibitor, sphingosine, blocks normal compaction at the late 8-cell stage. ${ }^{3)}$ In addition, Pey et al. reported that intracellular free calcium is involved in compaction and in relocation of E-cadherin and calmodulin. ${ }^{4}$ When 4-cell embryos were treated with $\mathrm{DiC} 8$, a PKC agonist, the embryos induced premature compaction within 30 min. ${ }^{11)}$ At this time it was also shown that $\beta$-catenin associated with E-cadherin was phosphorylated on serine/threonine residues and localized into the regions of cell adhesion and that the alpha isotype of PKC became enriched at the region of cell-cell contact.

Recently, it was reported by Goval and Alexandre that tyrosine kinase inhibitor, genistein, induces 4-cell arrest but cannot block the compaction. In other words, genistein causes a premature 4-cell compaction, so they suggested that

\section{(B) Calyculin A-treated 2-cell}
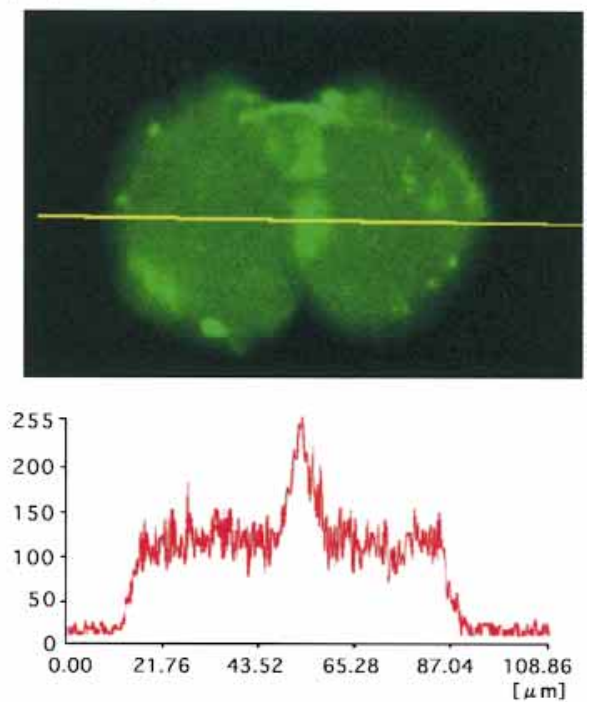

Fig. 5. E-Cadherin Distribution before or after Calyculin A Treatment

Two-cell embryos were incubated with or without $2 \mathrm{~nm}$ calyculin A for $24 \mathrm{~h}$. The embryos were immunostained with monoclonal E-cadherin antibody. E-cadherin distribution was analyzed by laser scanning confocal microscopy. The uniform distribution was observed in control two-cell (A) but enriched distribution at the plasma membrane of adjoining blastomeres was observed in calyculin A-treated embryos (B). 
the "compaction" takes place according to a biological clock. ${ }^{9}$ ) Their results demonstrate that very complicated interactions with some phosphorylation/dephosphorylation pathways control the cell-flattening phenomenon at the 8-cell stage according to a biological clock. In other words, the compaction is independent of the normal progress through the third and fourth cell cycles and that the compaction takes place according to a biological clock.

In the present study, we showed that Ser/Thr phosphatase inhibitor, calyculin A, caused 2-cell arrest, and that simultaneously, it also induced 2-cell flattening. A key molecule in compaction, E-cadherin, was relocalized in 2-cell embryos without transcriptional inductions by the addition of calyculin A. These facts may imply that the "compaction" is mainly regulated by the redistribution of E-cadherin into the cell-cell adjoining region. The relocation of E-cadherin may be regulated by the dephosphorylation of a still unknown molecule which seems to be a common substrate of both a PKC and a calyculin A-sensitive phosphatase. Further studies are necessary in order to clarify what kind of molecule is a substrate of calyculin A-sensitive phosphatase, and which type of $\mathrm{PKC}$ is concerned with the compaction.

\section{REFERENCES}

1) Cohen P., Annu. Rev. Biochem., 58, 453-509 (1989).

2) Pratt H. P. M., Surani M. A. H., Cell, 26, 279-292 (1981).

3) Winkel G. K., Ferguson J. E., Takeichi M., Nuccitelli R., Dev. Biol., 138, 1-15 (1990).

4) Pey R., Vial C., Schatten G., Hafner M., Proc. Natl. Acad. Sci. U.S.A., 95, 12977-12982 (1998)

5) Ohsugi M., Ohsawa T., Semba R., J. Exp. Zool., 265, 604-608 (1993).

6) Pauken C. M., Capco D. G., Mol. Reprod. Dev., 54, 135-144 (1999).

7) Vestweber D., Gossler A., Boller K., Kemler R., Dev. Biol., 124, 451456 (1987).

8) Takeichi M., Development, 102, 639-655 (1988)

9) Goval J. J., Alexandre H., Eur. J. Morphol., 38, 88-96 (2000).

10) Smith G. D., Sadhu A., Mathies S., Wolf D. P., Dev. Biol., 204, 537549 (1998).

11) Pauken C. M., Capco D. G., Dev. Biol., 223, $411-421$ (2000).

12) Kidder G. M., McLachlin J. R., Dev. Biol., 112, 265-275 (1985). 